\title{
"ERGONOMICS AS BASIS FOR A DECISION SUPPORT SYSTEM IN THE PRINTING INDUSTRY"
}

\author{
Mateo, Manuel. \\ Profesor del Departamento de Organización de Empresas \\ Universitat Politècnica de Catalunya
}

\section{Alvarez-Casado, Enrique}

Centre Específic de Recerca per a la millora i Innovació de les

Empreses -

Universitat Politècnica de Catalunya - Barcelona (Spain)

Mondelo, Pedro R.

Profesor titular del Departamento de Organización de Empresas

Universitat Politècnica de Catalunya

\section{Tarral, Marc}

Centre Específic de Recerca per a la millora i Innovació de les Empreses

INTRODUCCIÓN

Para que cada día por la mañana podamos comprar el periódico, es necesario que durante la noche anterior éste se imprima en una planta impresora. Además, a menudo el periódico no se vende tal cual, porque contiene diferentes suplementos como publicidad, ofertas o regalos. El presente trabajo se centra en una de estas plantas impresoras de última generación, donde cada noche se imprimen cinco periódicos diferentes, con un total de más de 400.000 ejemplares diarios distribuidos por España y Francia. El trabajo debe realizarse en un corto espacio de tiempo, desde que se da por finalizada la edición diaria hasta que las furgonetas recogen los paquetes de diarios para repartirlos por quioscos y librerías. Esto requiere que los procesos productivos estén perfectamente definidos para evitar que las furgonetas salgan con demoras y la empresa propietaria del periódico reclame penalizaciones a la planta impresora por retraso en la entrega.

Dentro de estos procesos productivos algunas de las operaciones de tipo manual adquieren especial importancia, especialmente en aquellos días en que se incluyen varios suplementos y folletos publicitarios. Para minimizar la cantidad monetaria de penalizaciones a pagar por la empresa impresora se ha diseñado un sistema para la toma de decisiones sobre el número de trabajadores necesarios para la inserción de los elementos de publicidad que acompañan a los diarios.

Por ello, en primer lugar se presenta la planta objeto de estudio y las principales etapas de su proceso productivo: el almacenaje, la impresión, la inserción de publicidades y la expedición. A continuación, se estudia con más detalle el proceso de la parte del Cierre, entendido este 
como el final del proceso donde se empaquetan los periódicos y se insertan las publicidades, para posteriormente analizar las posibilidades de mejora continua en esta planta. Así pues, la inserción y la expedición serán las actividades objeto de estudio de la fábrica.

La planta tiene máquinas de última generación, pero no presentaba unos buenos resultados de rendimiento porque los procesos estándares no estaban bien definidos, especialmente en el área del Cierre. Dado el carácter lineal del proceso del sistema productivo y el limitado tiempo para realizar todas sus operaciones, el rendimiento del Cierre limita el rendimiento global de toda la planta y no permite absorber un incremento de producción de la etapa anterior en las Rotativas, y cumplir a la vez con los plazos de entrega.

Esta posición entre el flujo Push de las Rotativas, que tienen un proceso optimizado desde la creación de la planta, y el flujo Pull, ya que con una puntualidad muy estricta las furgonetas deben salir a repartir periódicos, y esto obliga a la empresa de la planta a mejorar significativamente sus resultados en este ámbito para seguir siendo competitiva y conseguir nuevos clientes. El respeto a los plazos de entrega es clave en este sector, aunque desafortunadamente no es fácilmente controlable por la empresa, pues cada noche se trata de una producción diferente con un panel de opciones importante, sin que se pueda prever con suficiente antelación qué se va a incluir (insertar) en la próxima edición.

Por todo ello, la empresa decidió realizar un estudio completo de su proceso productivo en el área de Cierre para conocer con precisión las diferentes posibilidades de producción en su cuello de botella y, en especial, la gestión del personal necesario para cubrir esta tarea. Para ello ha sido necesario definir cada etapa del proceso, los estándares de trabajo y los tiempos unitarios de cada tarea.

El resultado del estudio es la creación de un sistema de información que sirva para el análisis de costes del proceso de Cierre y la toma de decisiones adecuadas para el cumplimiento de los objetivos de producción. Esto permite al director de producción determinar con precisión el personal temporal a contratar cada noche para el "cuello de botella" que se genera en el sistema de inserción manual de publicidad y suplementos.

La herramienta desarrollada, que determina las necesidades en recursos humanos, permite anticipar y adaptarse a las dificultades que antes se descubrían durante la producción. Todo esto, se ha desarrollado en el marco de un proceso de mejora continua en el Cierre que pretende aumentar el rendimiento de la planta implantando principios de Lean Management.

DESCRIPCION DE LA EMPRESA Y ELL PROCESO PRODUCTIVO

En la planta impresora se observan cuatro partes principales. La primera, que es el Almacén, contiene las bobinas de papel, las tintas y el resto de materias primas así como residuos del proceso de impresión. La segunda es la Pre-impresión donde se reciben las noticias de los periodistas y se elaboran las planchas que se colocan sobre los cilindros de las rotativas. En la tercera, las Rotativas, se imprimen, ensamblan y pliegan las páginas. Finalmente, en la cuarta parte, llamada Cierre, se recibe los periódicos impresos, se inserta las publicidades o suplementos, se empaquetan y se reparten entre las furgonetas que esperan en el muelle. $A$ cada ruta de distribución le corresponde una furgoneta con un lugar y una hora de recepción contratada con el cliente. La operación de obtención de los periódicos para insertar automáticamente otros documentos dentro de ellos se llama encartado. 
El proceso productivo de la planta está compuesto de un conjunto de etapas. Las primeras, consisten en el diseño, la digitalización, la filmación, la prueba de color, el montaje y la preimpresión (obtención de planchas para la impresión), se desarrollan antes de iniciar la impresión de los ejemplares en las rotativas. A continuación, se imprime el periódico en las rotativas (grandes cilindros). Una vez el papel ya incluye el contenido esperado se realiza el plegado de las hojas, el encartado automático de suplementos y el acabado, donde se hace el encartado de forma manual. La etapa de inserción manual es la más cotosa en términos de tiempo y personal porque se debe contratar personal de una empresa externa para hacer estas operaciones manuales. La última etapa consiste en la expedición hacia las diferentes playas en el muelle de expediciones, para el envío de los periódicos a cada punto de venta.

La capacidad productiva nominal de las rotativas es de 700.000 ejemplares de 112 páginas en un solo tramo de 8 horas de trabajo, lo que equivale a 28.000 millones de páginas al año. Cada una de las rotativas permite una velocidad de impresión de unos 85.000 ejemplares a la hora y una velocidad de encarte automático de 45.000 por hora (inclusión de suplementos y publicidades), tiempo en el que también se pueden clasificar hasta 12.000 paquetes de ejemplares. La maquinaria es de última generación y tiene capacidad de adaptación automática a formatos variables, con capacidad de graparlos y efectuar un corte para acabados de mayor calidad. Los almacenes están totalmente robotizados.

Un sistema de mando estructurado de forma modular Mailroom Production Control (MPC) enlaza en red todos los procesos parciales y asegura que las secuencias sean correctas. Este sistema de control permite una visión central y actualizada de todas las etapas del proceso y un flujo de material óptimo. Los productos iniciales (producto principal, o sea periódico, y eventualmente hasta dos productos adicionales, o sea suplementos) son conducidos de forma sincronizada desde el sistema de retención y almacenaje FlexiRoll al sistema de encarte. Además de estos productos iniciales, se pueden añadir cuatro encartes más.

Una vez salen los periódicos de las rotativas, los diferentes elementos que componen el área de Cierre son:

- Los Flexirolls son discos que actúan de buffer, sobre los cuales se enrollan los periódicos para producciones futuras o para sincronizar un encartado.

- Las Encartadoras, que son cuatro, efectúan la operación de encartado, es decir, reciben el producto principal, lo abren y pueden insertar dentro hasta 2 suplementos de manera automática y 4 de manera manual.

- Los Stackers o apiladores crean paquetes de periódicos y les ponen una protección (mediante flejes y papel kraft) y un código de barras.

- El Sorter es una cinta transportadora que, leído el código de barras de un paquete, lo distribuye hacia uno de los muelles de expedición.

La impresión de los periódicos puede seguir dos recorridos en la planta:

- Sacar directamente el producto por los apiladores (Stackers) y mandarlo a la cinta de transporte (Sorter) que dirige cada paquete de este producto a un muelle, donde le espera una furgoneta.

- Realizar una operación de almacenamiento temporal (Flexirolls) para coordinar su posterior encartamiento de publicidad, antes de mandarlo al Sorter para su expedición. 
El proceso de encartado corresponde a la alimentación productos publicitarios o suplementos en el flujo de la producción para que éstos sean insertados dentro del producto principal que sale de las rotativas. Estos suplementos o encartes pueden variar según el punto de venta, es decir, el área geográfica elegida por el departamento marketing de la empresa anunciadora. Así, un mismo periódico se puede producir simultáneamente para convertirse en varios productos finales distintos.

Para insertar los suplementos existen dos opciones de producción posibles:

1. Encartado automático durante la noche (stock). Se utiliza en caso que el encarte se pueda enrollar en discos. El encarte se almacena durante el día y espera en discos hasta la impresión del periódico durante el turno de noche para ser insertado de manera automática en la encartadora. Una vez lanzada la orden de producción del encarte; las líneas de periódicos y encartes se cruzarán en la Encartadora o Proliner donde se hace la inserción del encarte dentro del periódico. En este momento, el producto ya está listo para ser empaquetado y distribuido.

2. Encartado manual durante la noche (Just-In-Time). Esta producción es la más utilizada porque se utiliza para todos los encartes que no se pueden almacenar en discos, por razones de formato, que sea materia frágil... En este caso la empresa recurre a personal externo que se contratan según las necesidades de cada día: en función del tipo de encarte, la dificultad y el número de ejemplares. Este personal, que a veces representa hasta un $60 \%$ del personal total dedicado a la producción en planta, inserta directamente en la encartadora, teniendo cada incidencia repercusiones directas en toda la línea de producción. Su trabajo consiste en preparar los encartes publicitarios que llegan en palets protegidos, flejados o en cajas e insertarlos en la línea de carga conectada con la Proliner.

En el caso del encartado automático, el proceso es más fiable porque los operarios intervienen mucho menos, aunque está limitada la velocidad a la cual se encarta por el tipo de encarte y la calidad del periódico que sale de las rotativas.

En el caso de encartado manual durante la producción de noche los ejemplares de periódico llegan automáticamente por una línea hasta la Encartadora. Uno o varios operarios aprovisionan de encartes y tienen que aguantar el ritmo impuesto por la línea. Por ejemplo, si un encarte se atasca porque no ha sido bien preparado o el aprovisionamiento de encartes no es suficiente rápido, toda la línea se para hasta que el problema se haya solucionado. Este proceso de encartado es clave porque es cuello de botella, además de tener el coste en personal más elevado de la planta. Además, para estos puestos de trabajo el personal empleado es temporal, con muy poca experiencia y una formación mínima para cumplir sus tareas.

Inicialmente, no se sabía evaluar el número de operarios necesarios para realizar el insertado manual ni los rendimientos alcanzables de las encartadoras en función de los tipos de encartes. Por eso, se decidió de crear una clasificación de los encartes para definir, mediante un estudio de tiempos y de las características de los encartes, las necesidades en personal para realizar el proceso de inserción manual. No se conocían tampoco con precisión los recursos necesarios para preparar la producción. Esto generaba muchas paradas de la línea en el momento de insertar. 


\section{Situación de partida y objetivos}

Dado que en el área del Cierre no se disponía de un proceso estandarizado, el primer objetivo fue definir cada operación en términos de tiempos y personal necesario. Este objetivo se materializó en las siguientes fases:

- Definición de los procesos de preparación de las máquinas.

- Determinación de los tiempos unitarios de las operaciones de producción.

- Determinación de las necesidades de personal para realizar la preparación de máquinas y la producción.

- Determinación de las necesidades de personal externo para efectuar las operaciones de encartamiento manual.

- Propuesta de acciones de mejora del proceso y puesta en marcha.

La cantidad de personal externo de la empresa se contrata en función de las necesidades de cada noche para ejecutar las tareas más simples y repetitivas del Cierre. La necesidad de comunicación permanente con este personal obliga a que un encargado vigile la ejecución de las tareas. Este personal realiza las siguientes tareas en el turno de noche:

- Descargar los palets de los encartes (elementos a incluir dentro de cada ejemplar de periódico) y prepararlos sobre las mesas frente a las Encartadoras.

- Cargar las líneas Stream Feeder de las Encartadoras en encartes (proceso de inserción manual).

- Clasificar los ejemplares del periódico, eventualmente encartarlos, cuando es necesario para completar los envíos.

- Embolsar periódicos que se distribuyen por envío especial.

\section{Proceso de inserción manual}

\subsection{Proceso global de inserción manual}

El proceso de inserción manual es el cuello de botella del área de Cierre y el más complejo porque tiene que adaptarse al acondicionamiento de los encartes que mandan los proveedores, está muy controlado por los clientes y supone el coste en personal más elevado de la empresa. La inserción manual de encartes en la Proliner consta de las siguientes operaciones:

- Preparación de los encartes. Consiste en quitar las protecciones de los encartes, es decir, preparar los encartes para permitir una inserción óptima. Esta actividad depende de la gran variabilidad de los tipos de condicionamientos usados por los proveedores.

- Inserción manual en la máquina. Consiste en encuadrar los encartes ya preparados y depositarlos de manera adecuada en el Stream feeder.

Los jefes de producción no sabían qué cantidad de recursos se debía asignar a la inserción de publicidad. Para afrontar este complejo problema se ha realizado un estudio completo del proceso para estandarizarlo y automatizarlo. Los resultados obtenidos se muestran en los siguientes apartados.

\subsection{Cálculo del tiempo tipo y ciclo normal de la inserción manual}


A fin de conocer los tiempos de producción del proceso de inserción manual, se ha realizado un estudio de tiempos. Para determinar el Tiempo Normal se ha aplicado el sistema de tiempos predeterminados MTM-2, a partir de un conjunto de observaciones directas efectuadas durante un mes. Los resultados obtenidos tienen una fiabilidad de un $90 \%$.

A los tiempos normales calculados con MTM-2 se les ha aplicado un coeficiente de descanso (columna K) que oscila entre el $11 \%$ y el $16 \%$, según el elemento considerado, y que incluye los siguientes factores:

- Esfuerzo ejercido: Según el peso de los materiales manipulados, la frecuencia con que se realiza el esfuerzo y la parte del cuerpo afectada (extremidades superiores).

- Postura de trabajo: Normalmente de pie, con desplazamientos libres con o sin carga, implica un grado ligero con un nivel alto.

- Ciclo breve: Trabajo muy repetitivo en los músculos de los dedos, manos y brazos.

- Concentración: Atención exigida en los movimientos ejercidos para la colocación de los encartes en el alimentador. Se considera un grado ligero a nivel alto (3 puntos).

- Monotonía: Efectuar un mismo trabajo repetitivo se considera con un grado ligero de nivel alto (2 puntos).

- Ruido: El trabajo se efectúa en un taller con ruido continuo. Por eso, se considera un grado mediano a un nivel bajo ( 3 puntos).

\subsection{Determinación de las limitaciones ergonómicas del proceso}

Se pretende determinar los límites del proceso de inserción manual en términos de cantidad manipulable por ciclo, es decir, altura de mano (en centímetros) y peso manipulable.

Altura de mano: Corresponde a la distancia entre la primera articulación del pulgar y la segunda del índice, los dedos aplicando una presión suficiente sobre el paquete de publicitarios para manipularlo. Este proceso puede ser realizado tanto por hombres como mujeres, pero tal como señala la norma UNE-EN ISO 15537:2004, debemos centrarnos en las medidas de la mano de mujeres tipo pequeño. A continuación, se presentan en la figura 1 las medidas de la mano al nivel mundial de mujeres. 


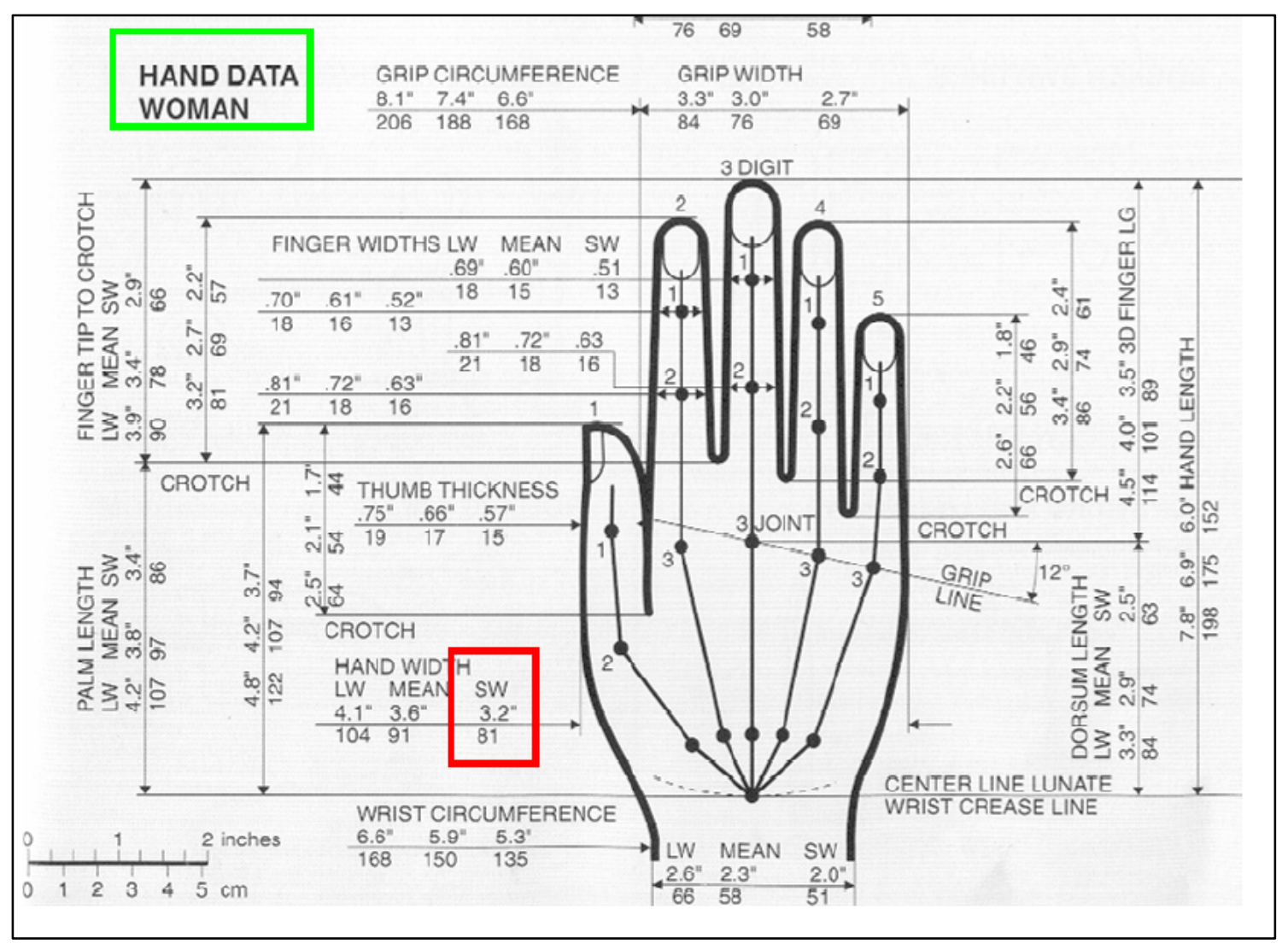

Figura 1: Norma de los datos antropométricos de la mano de mujeres (norma UNE-EN ISO 15537:2004)

Se ha aproximado la distancia que interesa medir a la distancia entre el inicio de la palma de la mano hasta la extremidad del pulgar. Con esta distancia, que se puede aproximar a 10,7 cm, se podrá calcular el límite en términos de número de ejemplares ideal a manipular por ciclo.

Límite de peso: Por otra parte, se ha determinado el límite en términos de peso manipulable por ciclo para que no conlleve ningún riesgo para la salud del operador. Del mismo modo se ha considerado que este proceso puede ser efectuado por un hombre como por una mujer.

Gracias a un programa del grupo de profesorado de ergonomía del CERpIE del Departamento de Organización de empresas de la Escuela Técnica Superior de Ingenieros Industriales de Barcelona (ETSEIB), se introducen los parámetros siguientes:

- Tiempo de ciclo.

- Altura de recogida de la carga.

- Altura de descarga de la carga.

- Duración del proceso.

- Distancia que recorre el operador con la carga.

- Desviación angular del cuerpo durante el ciclo.

Como se puede ver en la figura 2 El programa indica que, dados los valores de estos parámetros, se puede manipular una carga de hasta $4,1 \mathrm{Kg}$ por ciclo sin que el operador tenga riesgo crítico para su salud. Ahora toca determinar en función de las características principales de los encartes, la paginación y el gramaje, cuántos encartes puede manipular un operario sin sobrepasar una de estas dos limitaciones. 


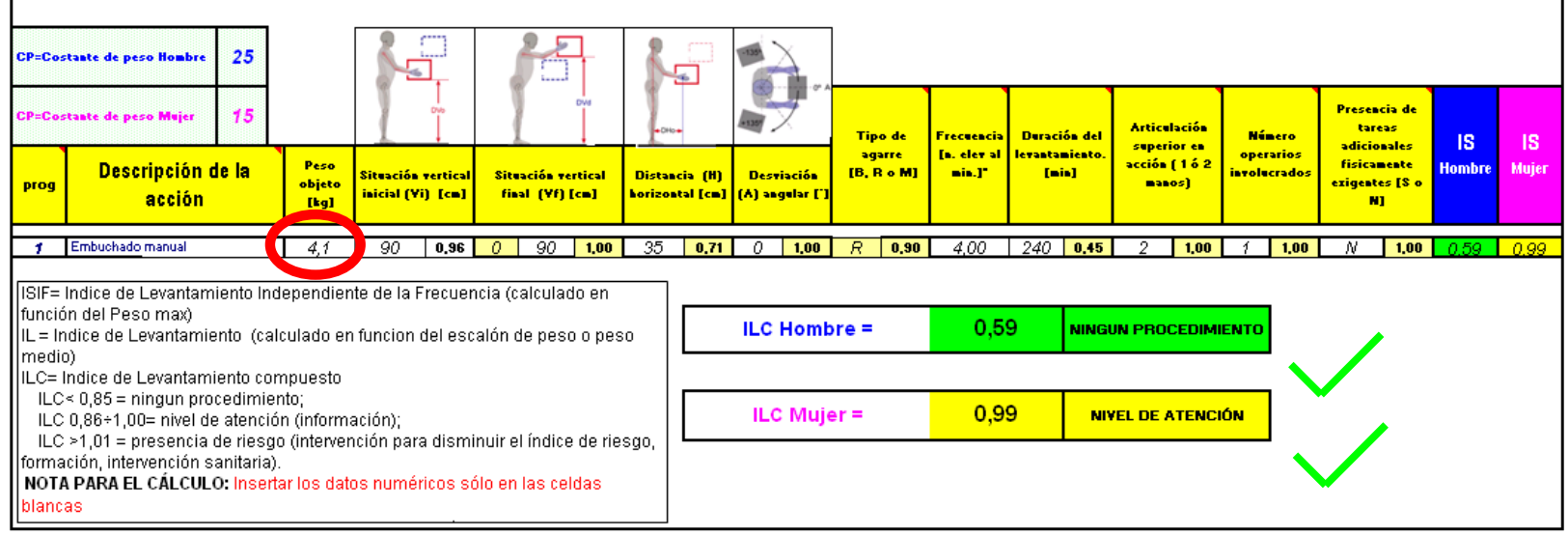

Figura 2: Programa de cálculo del nivel de riesgo para tareas múltiples

\subsection{Clasificación de los encartes}

A continuación, se evalúa para cada situación de manera precisa la cantidad máxima de encartes que respetan estas limitaciones. El problema para determinar estas cantidades en función de las características de los encartes es doble:

- Por un lado, para el límite de altura de mano no existe una base de datos que permita conocer el grosor de una hoja de encarte a partir de su gramaje, que es la única información técnica que nos transmite el proveedor. Como esta base de datos es indispensable, se deberá crear efectuando un análisis de grosor de todos los encartes recibidos en el año 2008 en el laboratorio con un palmer.

- Por otro lado, existen grandes dudas de la validez de la información que nos da el proveedor sobre el gramaje teórico porque se compra a peso y el gramaje no toma en cuenta el peso añadido por la tinta. Simultáneamente, se realiza una base de datos de gramaje "real" gracias a una balanza de precisión.

Se ha realizado un estudio sobre 87 encartes, de los cuales se ha descartado 18 por demasiada diferencia entre los pesos real y teórico del encarte. Por lo tanto, quedan 69 encartes, que se clasifican en 14 grupos en función del gramaje teórico.

En la figura 3 se presentan los valores teóricos por parte del proveedor y los experimentales determinados: grosor media, espesor encarte y peso real y sus valores medios. 


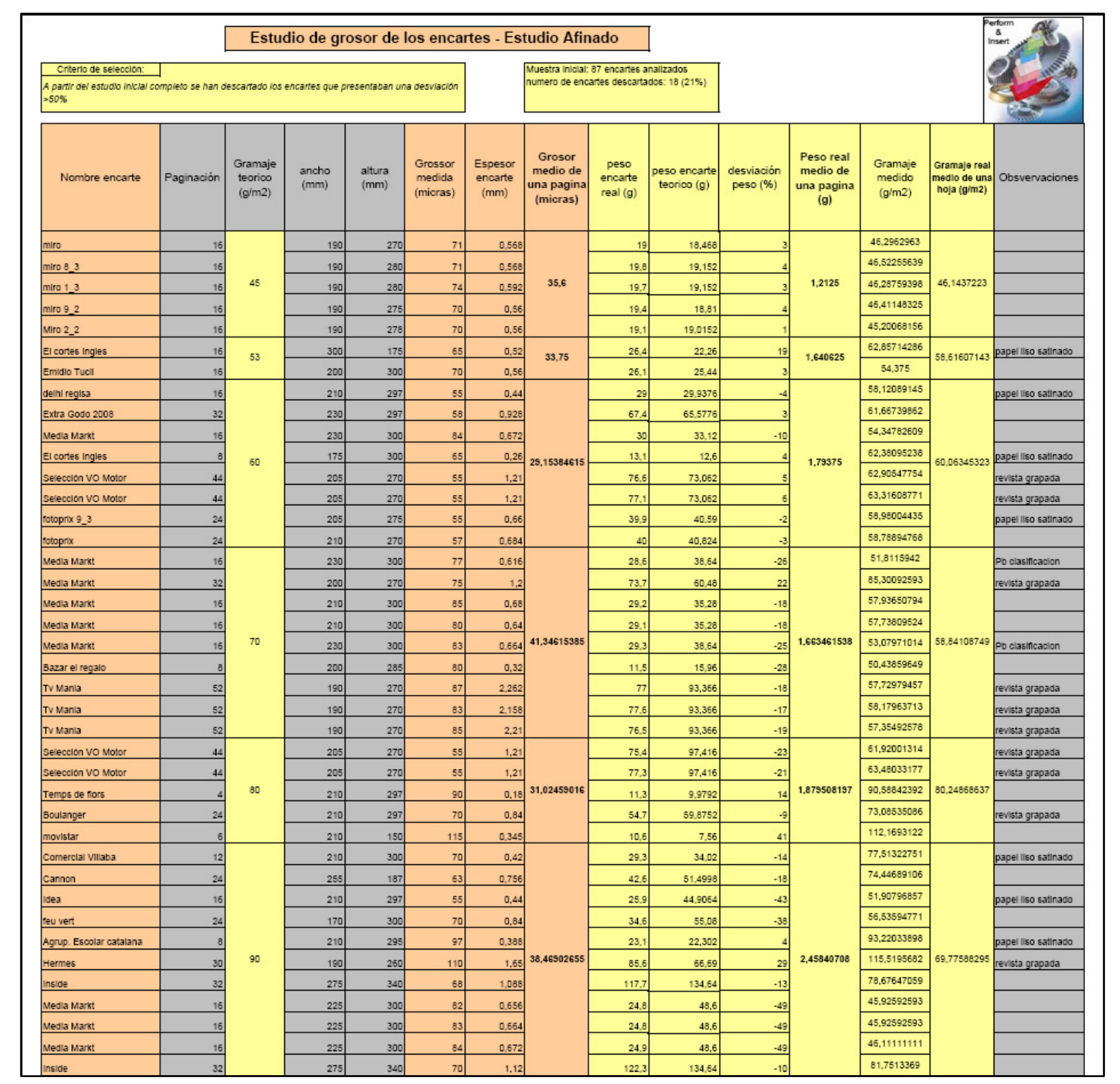

Figura 3: Extracto del estudio afinado de grosor y gramaje real de los 69 encartes

El objetivo de este estudio es saber a partir de las informaciones que da el proveedor, gramaje teórico y paginación, el peso real del encarte y su espesor real, y así determinar la cantidad que podrá manipular e insertar un operario por ciclo. A partir de esta base de datos, se hace un análisis gráfico de los resultados con regresiones lineales para obtener una tabla de valores de grosor y gramaje en función de cada encarte.

La correlación entre los gramajes teóricos indicados por parte de los proveedores y los gramajes reales medidos en el laboratorio de la empresa tiene un coeficiente $R^{2}=0,90$. Esto valida la hipótesis que la tinta influye, entre otros factores, en el peso final del encarte. Para determinar la cantidad máxima de encartes manipulables respetando el límite por peso, se toma como referencia el gramaje medido ajustado por la regresión lineal.

Mediante regresión lineal, se determina el peso medio de una página de encarte en función de su gramaje teórico, con un coeficiente de correlación $R^{2}=0,86$. Gracias a esta regresión lineal 
se conoce con más exactitud el peso real de una página de encarte a partir del gramaje anunciado por el proveedor. Esta información sirve también para conocer las cantidades manipulables respectando el límite de peso del proceso de inserción manual.

De forma similar, se determina el grosor medio de una página de encarte en función de su gramaje teórico, donde el coeficiente de correlación de la regresión lineal es $R^{2}=0,80$. Así, se puede conocer con más exactitud el grosor real de un encarte a partir del gramaje anunciado por el proveedor. Esta información nos interesa para poder determinar la cantidad de encartes manipulables por parte del operario, respectando el límite de agarre de la mano.

A partir de las ecuaciones de las regresiones lineales, una base de datos que parte del gramaje teórico dado por el proveedor obtiene el grosor de una página y el gramaje real. La base de datos experimentales inicial con 14 grupos se ha extrapolado a 35 grupos de gramaje diferentes.

\subsection{Determinación del personal necesario}

La tabla de las cantidades máximas manipulables por el personal respetando los límites ergonómicos ha sido calculada para 2 tipos de formatos: DINA4 y DINA5 (los más frecuentes). A partir de estas cantidades, se determina el número de operarios necesarios para aguantar el ritmo de trabajo en una Proliner en función de diferentes velocidades mecánicas.

Para facilitar el personal necesario destinado a realizar el encarte manual se ha desarrollado una herramienta Excel de tipo "Macro" que es un sistema de ayuda a la toma de decisiones. Permite saber el personal temporal que se debe contratar en función de los objetivos de producción que se fijan y su cálculo se basa en:

- Una base de datos experimentales.

- El estudio de tiempo de ciclo del proceso de inserción manual.

- El estudio de límites ergonómicos del proceso de inserción manual.

\section{Proceso de preparación de los encartes}

\subsection{Definición del proceso de preparación de los encartes}

Conocido el personal necesario para realizar la alimentación de los Stream Feeders de las encartadoras Proliner, así como las cantidades ideales a manipular al nivel ergonómico, se debe determinar cuánto tiempo es necesario para preparar los encartes. Este proceso consiste entonces en quitar todas las protecciones que lleva el pallet de encarte (plásticos retractilados, flejes, cajas, etc...) y preparar paquetes del tamaño óptimo a nivel ergonómico. La gran dificultad viene del hecho que cada proveedor manda sus encartes según sus criterios de condicionamiento.

Para determinar el tiempo unitario de preparación adecuada de los encartes se ha realizado un estudio de tiempos para identificar los diferentes tiempos unitarios estándares en función de las características de condicionamiento del encarte (protegido, flejado, retractilado, encajado, etc.). 


\subsection{Determinación del personal necesario}

Estos estudios han permitido diseñar un programa de ayuda a la toma de decisiones, similar al de anterior de determinación de personal para el proceso de inserción, que evalúa el personal temporal necesario para preparar los encartes.

Así, se puede conocer con antelación de manera precisa las necesidades en personal mientras antes se recibían los encartes y sólo se preparaba justo antes de la producción de noche o se paraba la producción para incluir este proceso. Esta falta de organización y de planificación entrañaba muchos problemas de personal y perturbaciones de la producción. Con esta nueva herramienta se puede saber en función de la tirada a encartar (por ejemplo, 100.000), del número de pallets ( 5 en este ejemplo) y de la cantidad de encartes por paquete (100 ejemplares en este ejemplo) y de su tipo de condicionamiento (flejado, por ejemplo) el tiempo necesario para preparar todos los encartes (207 minutos o sea 3,45 horas con 2 operarios temporales). Esto se puede observar en la figura 4.

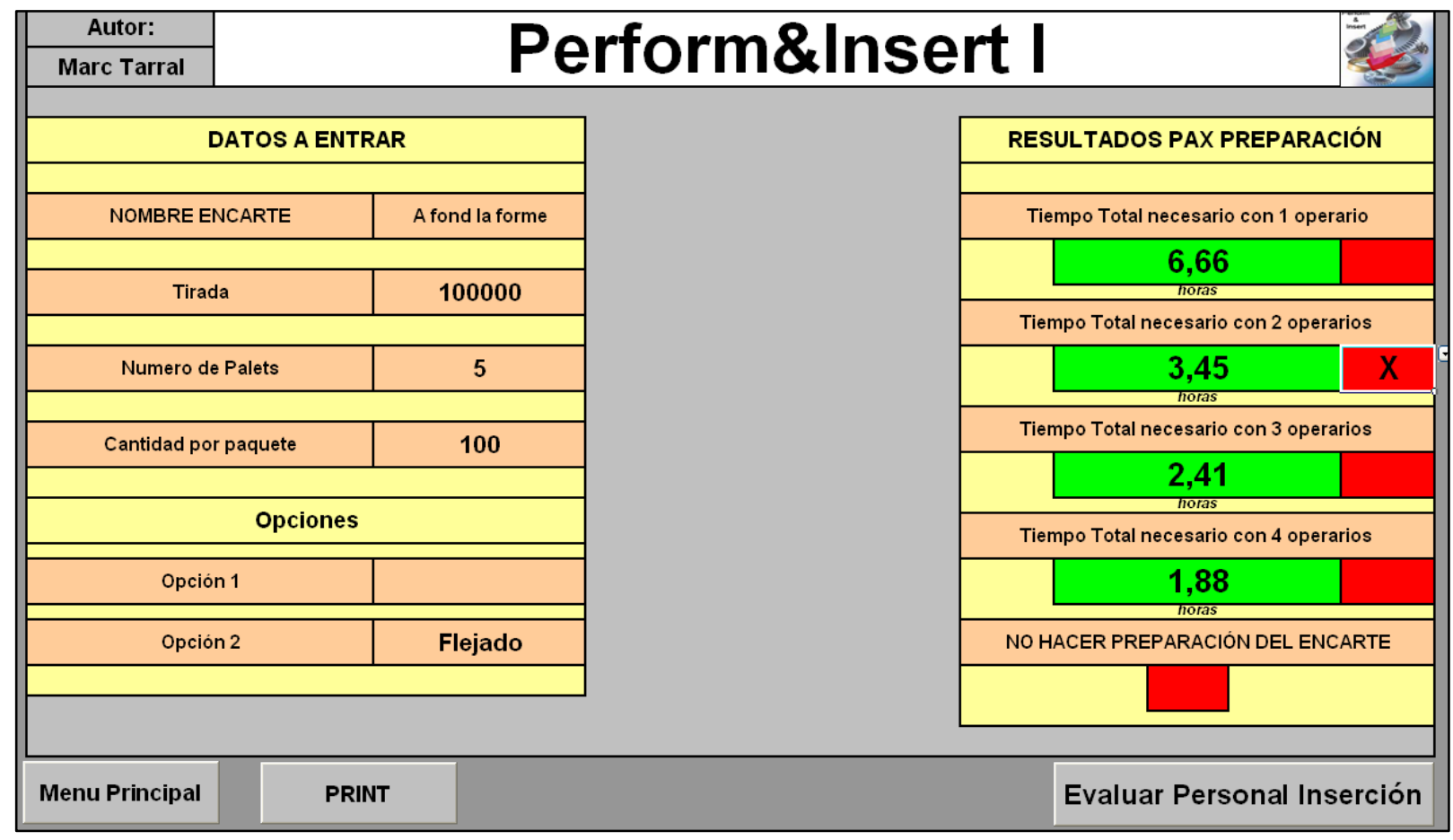

Figura 4: Ejemplo de cálculo de recursos en personal necesario para la preparación de un encarte

\section{Consecuencias en la organización del encarte}

\subsection{Redefinición del flujo}

Para resolver los problemas de inserción de encartes debido a la acumulación de tareas durante el turno de noche, se ha decidido cambiar el flujo de los encartes como se puede ver en la figura 5. 


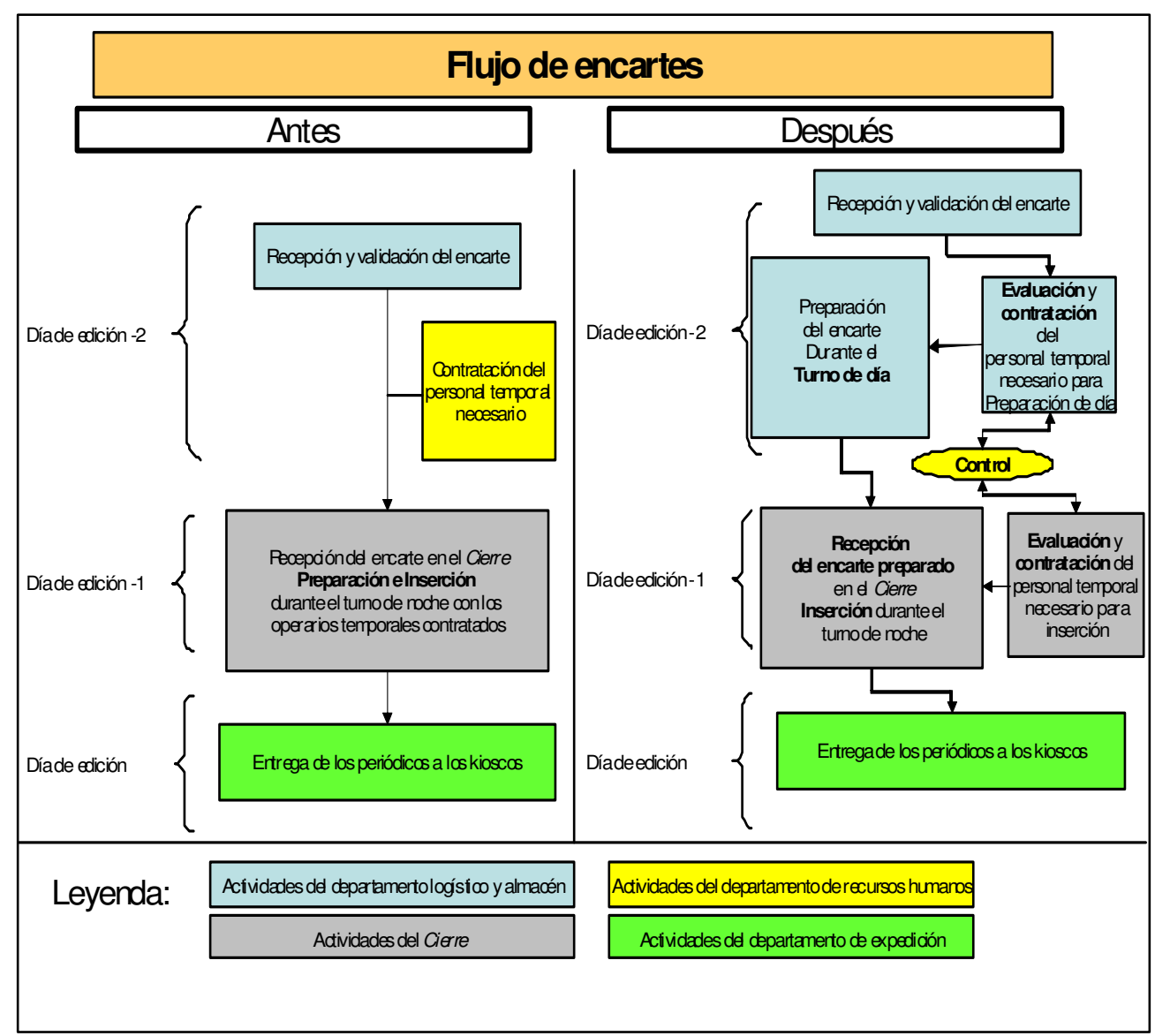

Figura 5: Redefinición del flujo de encartes

Para evitar atascos de producción asociados a la sobrecarga de trabajo en los encartes se ha decidido de realizar la operación clave de preparación de los encartes como mínimo un día antes de la inserción. Esta tarea ha sido imputada al departamento logístico, que debe recibir los encartes, validar su conformidad y prepararlos en condiciones óptimas para su inserción. Este cambio ha permitido:

- Lograr rendimientos superiores en los cuellos de botella que son las Proliners

- Reducir el coste de preparación, haciendo esta tarea de día con un personal que tiene un coste menor

- Conocer con precisión el coste total imputado a la inserción de cada encarte.

\subsection{Reorganización de la preparación de maquinas en casos extremos}

Hasta ahora, teóricamente existían 6 configuraciones de producción (según número de encartes manuales y automáticos) para los dos periódicos principales (3 para ambos), en que los maquinistas sobrepasaban los 60 minutos reservados a la preparación de maquinas antes de arrancar la producción (véase tabla 6). Además, en otros 4 casos los tiempos eran muy cercanos, 54 y 58 minutos. Para estos casos con la presencia de 2 o 3 encartes manuales, se ha decidido aprovechar la reorganización del flujo de encartes y adelantar la preparación de 
maquinas en estos casos "extremos" pero frecuentes. En realidad, la secuencia de tareas de preparación de la Proliner superaba los 80 minutos, lo que comportaba casi siempre retrasos importantes en la producción y la entrega de los periódicos.

La reorganización de la preparación de las Proliners ha comportado una reducción de los tiempos de preparación hasta que todas las configuraciones se preparan en menos de 60 minutos (límite antes que las rotativas empiecen a mandar los periódicos al Cierre).

Para cada caso de preparación se ha evaluado si era necesario preparar la totalidad o solamente una parte de encartes automáticos para cuadrar con el objetivo de la preparación de noche en 60 minutos teniendo siempre un pequeño margen de seguridad.

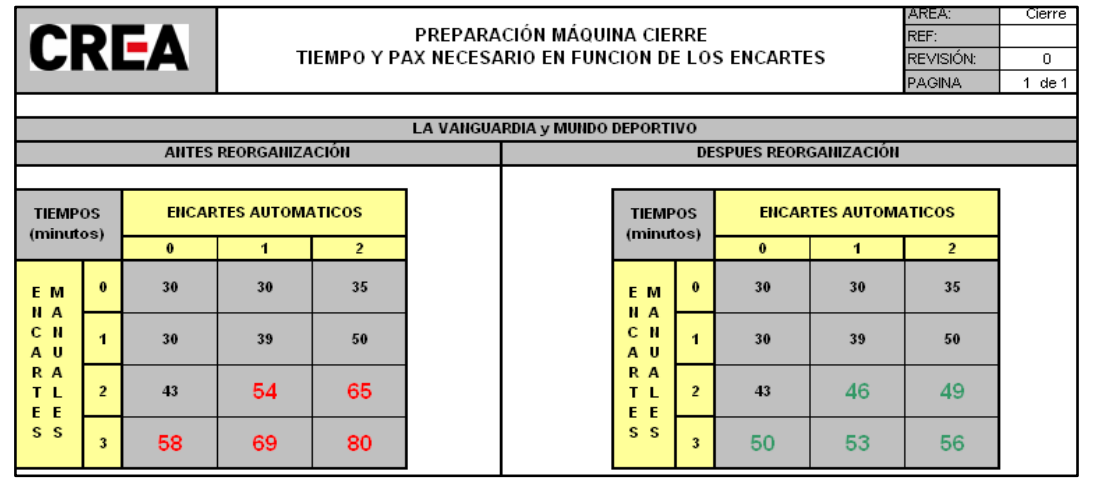

Antes (80min)

Después (56min)

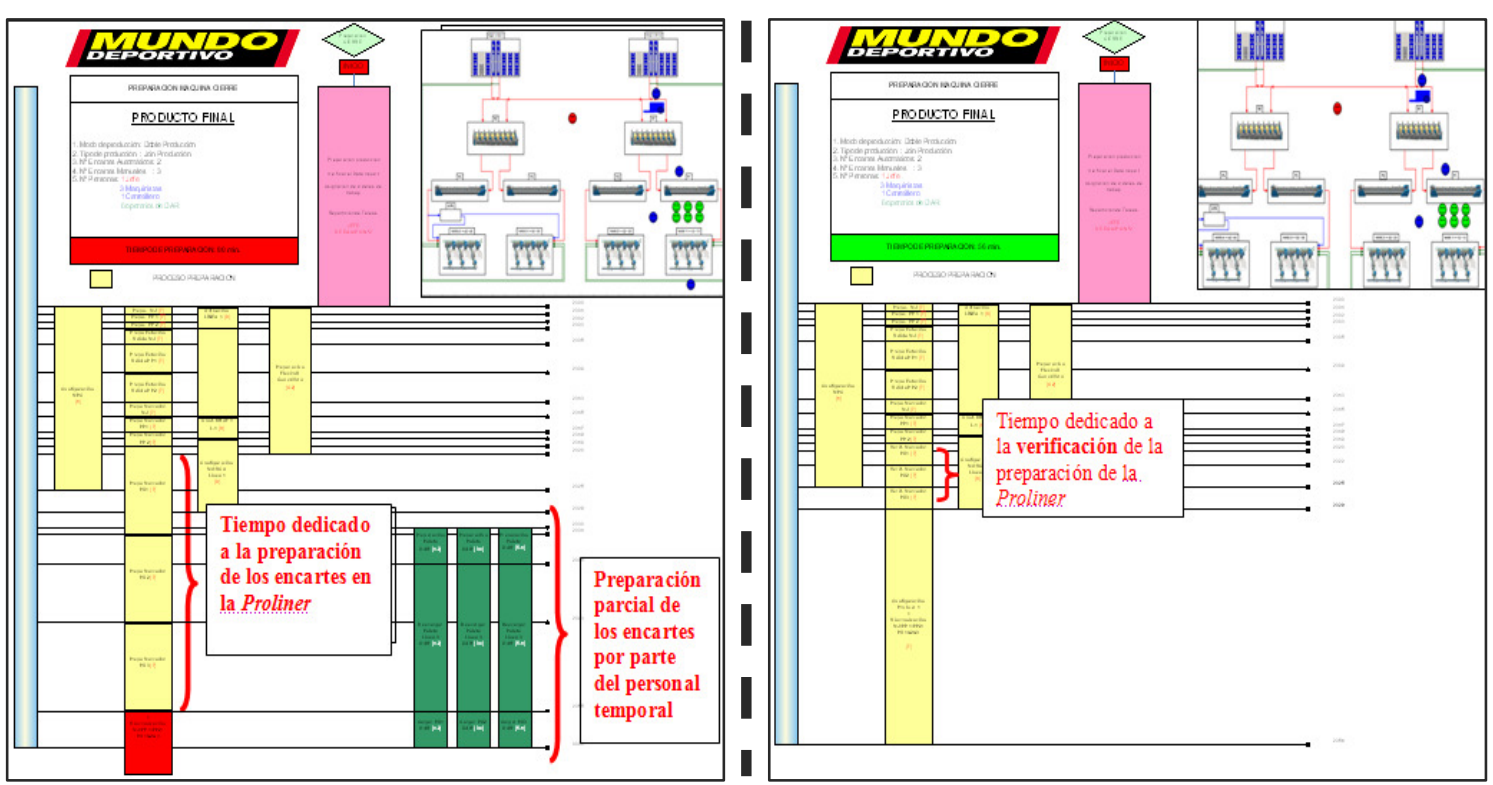

Tabla 6: Configuraciones de producción

\subsection{Influencia en la organización de la producción}

La repercusión de la utilización del sistema de ayuda a la toma de decisiones, combinado con las mejoras en el proceso en el encartado, ha sido inmediata en los resultados de la empresa.

- Evaluación de los rendimientos de las Proliners. El hecho de recibir un encarte bien preparado y tener el personal necesario ha permitido lograr un rendimiento más alto en este cuello de botella de la producción. La evolución del número de ejemplares por hora tratados lo avala (figura/datos). 


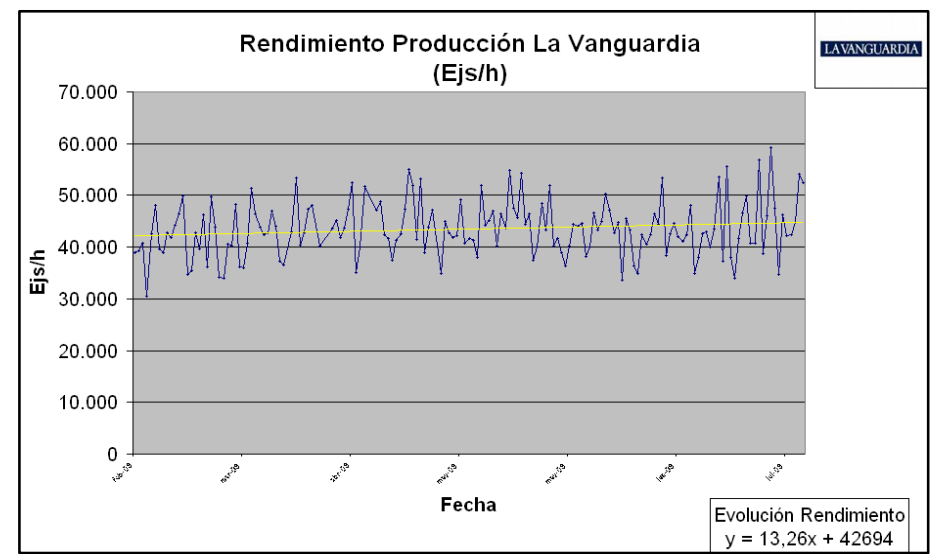

- Evolución del número global de paradas. La mejor planificación de las tareas a realizar en las Proliner, gracias al nuevo software, permite que se pueda observar más serenamente el funcionamiento de las máquinas y evitar paradas que anteriormente se hubieran producido.

- Evolución de los derroches de papel en la planta. Estos derroches (roturas de bobinas de papel,...) se producen por problemas relacionados con averías y atascos de las máquinas. Su reducción está directamente relacionada con que los encartes estén siempre preparados de manera adecuada.

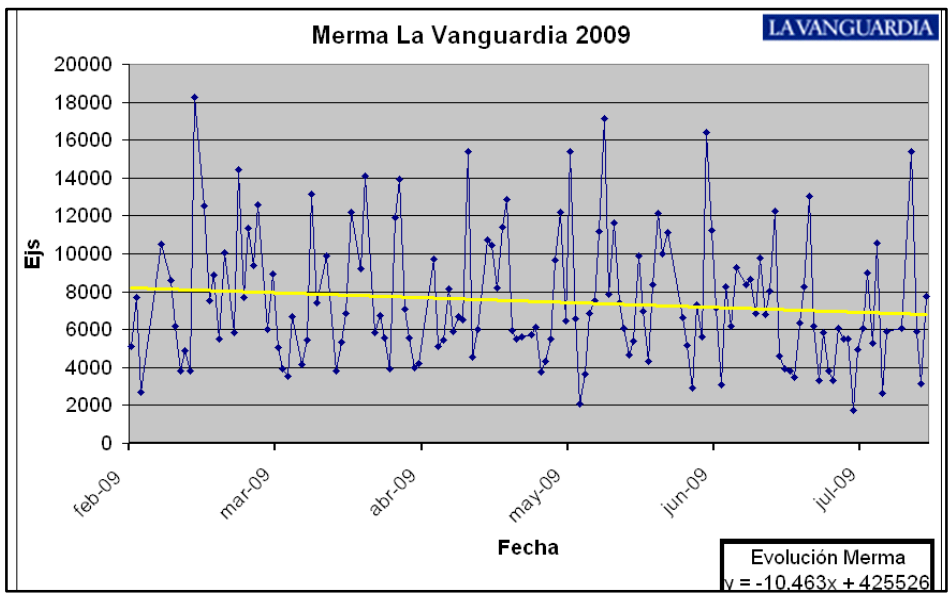

- Contratación de operarios en el encarte. Hoy en día se contrata un número de operarios ajustado a las necesidades con objetivos claros y definidos, mientras que antes solamente se evaluaba una cantidad necesaria y a menudo se añadía un margen para poder cumplir los plazos de entrega. A partir de octubre de 2008 se produce una reducción media de un $6 \%$ del número de horas efectuadas por operarios temporales en periodos de alta actividad. Esta reducción se produjo mientras el volumen de producción aumentaba con un nuevo periódico semanal de gran volumen.

\section{Conclusiones}

Este trabajo se ha realizado en una planta de impresión de una empresa que prevé crecer mucho en los próximos años, para lo cual debía aplicar cambios operativos para garantizar la calidad deseada y aumentar su capacidad de producción a fin de imprimir nuevos periódicos.

Para ello, se define la situación actual de la producción del Cierre para producir más de 500.000 ejemplares cada día. Se han analizado sus problemas y puntos débiles, uno de los cuales es el proceso de inserción manual de la Proliner, cuello de botella de toda la planta. Por ello, se ha realizado un estudio específico, que ha comportado: 
- El desarrollo de dos programas de ayuda a la toma de decisiones para determinar el personal temporal necesario en función de los parámetros de producción.

- Una reorganización del proceso de preparación de los encartes.

- Una redefinición del proceso de preparación de las Proliners.

- Un cambio del flujo logístico de los encartes publicitarios en la empresa.

Los estudios de ergonomía y de tiempos en la preparación e inserción de los encartes manuales han permitido ajustar el personal temporal a contratar en función de las características de los encartes, sus cantidades y las velocidades de producción que se quieran alcanzar. El Sistema de Ayuda a la Toma de Decisiones muestra el número de operarios necesarios para aguantar una cierta velocidad de inserción.

La redefinición del flujo de los encartes con la nueva etapa de preparación, antes de entregarlos totalmente preparados al Cierre, permite una reducción de las incidencias de producción en el momento de encartar. Para ello, se ha redefinido el proceso de "preparación de encartes", que se realiza en el almacén desde la llegada del encarte a la empresa.

La definición precisa del personal temporal necesario, junto a otras medidas complementarias, ha tenido varios efectos:

$>$ Reducción de costes de materiales (gastos de papel, gastos de paquetería y palets) a nivel de 58.300€/año

$>$ Aumento de productividad del $13 \%$ equivalente a un coste de oportunidad de 1.585.560€/año

$>$ Reducción de los tiempos de preparación y de los retrasos de producción equivalente a un ahorro de $52.500 €$ en extracostes

Reducción de los recursos en personal externo

\section{Agradecimiento}

Este trabajo se deriva en parte del proyecto "Programación de operaciones multicriterio con máquinas paralelas, en varias etapas, sin interrupciones ni almacenajes" (DPI2007-61371), financiado por el Ministerio Ciencia y Educación. 